\title{
Characterization of HIV-1 uncoating in human microglial cell lines
}

\author{
Zachary Ingram, Melanie Taylor, Glister Okland, Richard Martin and Amy E. Hulme*
}

\begin{abstract}
Background: After viral fusion with the cell membrane, the conical capsid of HIV-1 disassembles by a process called uncoating. Previously we have utilized the CSA washout assay, in which TRIM-CypA mediated restriction of viral replication is used to detect the state of the viral capsid, to study the kinetics of HIV-1 uncoating in owl monkey kidney (OMK) and HeLa cells. Here we have extended this analysis to the human microglial cell lines CHME3 and C2O to characterize uncoating in a cell type that is a natural target of HIV infection.

Methods: The CSA washout was used to characterize uncoating of wildtype and capsid mutant viruses in CHME3 and C20 cells. Viral fusion assays and nevirapine addition assays were performed to relate the kinetics of viral fusion and reverse transcription to uncoating.

Results: We found that uncoating initiated within the first hour after viral fusion and was facilitated by reverse transcription in CHME3 and C20 cells. The capsid mutation A92E did not significantly alter uncoating kinetics. Viruses with capsid mutations N74D and E45A decreased the rate of uncoating in CHME3 cells, but did not alter reverse transcription. Interestingly, the second site suppressor capsid mutation R132T was able to rescue the uncoating kinetics of the E45A mutation, despite having a hyperstable capsid.

Conclusions: These results are most similar to previously observed characteristics of uncoating in HeLa cells and support the model in which uncoating is initiated by early steps of reverse transcription in the cytoplasm. A comparison of the uncoating kinetics of CA mutant viruses in OMK and CHME3 cells reveals the importance of cellular factors in the process of uncoating. The E45A/R132T mutant virus specifically suggests that disrupted interactions with cellular factors, rather than capsid stability, is responsible for the delayed uncoating kinetics seen in E45A mutant virus. Future studies aimed at identifying these factors will be important for understanding the process of uncoating and the development of interventions to disrupt this process.
\end{abstract}

Keywords: Human immunodeficiency virus, HIV-1, Uncoating, Capsid, CA, Microglia

\section{Background}

After fusion of the viral membrane the conical capsid of HIV is released into the cytoplasm of the cell. This capsid contains approximately 1500 monomers of the viral capsid protein (CA) arranged in a hexameric lattice around the viral RNAs and associated proteins [1-3]. In order for infection to progress this conical capsid

\footnotetext{
* Correspondence: amyhulme@missouristate.edu

Department of Biomedical Sciences, Missouri State University, Springfield, $\mathrm{MO}$, USA
}

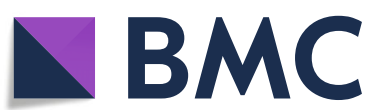

structure disassembles by a process called uncoating. During this time, the viral RNA is reverse transcribed into a double stranded DNA. The viral complex of nucleic acid and associated proteins also must traffic through the cytoplasm on microtubules [4-6]. This viral complex then gains access to the nucleus through a nuclear pore and the viral DNA is integrated into the chromosomal DNA to establish infection of a cell. Recent evidence suggests that CA and uncoating are at the crossroads of a complex interplay between these early steps of HIV replication. Some CA mutations can

(c) The Author(s). 2020 Open Access This article is licensed under a Creative Commons Attribution 4.0 International License, which permits use, sharing, adaptation, distribution and reproduction in any medium or format, as long as you give appropriate credit to the original author(s) and the source, provide a link to the Creative Commons licence, and indicate if changes were made. The images or other third party material in this article are included in the article's Creative Commons licence, unless indicated otherwise in a credit line to the material. If material is not included in the article's Creative Commons licence and your intended use is not permitted by statutory regulation or exceeds the permitted use, you will need to obtain permission directly from the copyright holder. To view a copy of this licence, visit http://creativecommons.org/licenses/by/4.0/. The Creative Commons Public Domain Dedication waiver (http://creativecommons.org/publicdomain/zero/1.0/) applies to the data made available in this article, unless otherwise stated in a credit line to the data. 
disrupt reverse transcription, but blocking reverse transcription also delays uncoating [7-10]. Disruption of protein interactions required for cytoplasmic trafficking affects the extent of uncoating [11-19]. Finally, determinants for nuclear import and integration site selection map to the CA protein [20-23], but the viral capsid is too large to enter the nuclear pore. Some amount of CA protein is proposed to associate with the viral complex after uncoating to facilitate integration, which has been detected in nuclear viral complexes [24-26]. Therefore, it is necessary to characterize the process of uncoating in order to fully understand the early events of HIV replication.

When, where, and how uncoating occurs is an area of active investigation and source of contention in the field. Currently there are two models for uncoating [27]. In the cytoplasmic uncoating model, uncoating occurs in the cytoplasm as the viral complex is trafficked toward the nucleus $[4,8,28-30]$. According to the nuclear pore model, uncoating occurs at the nuclear pore after docking of the intact capsid [31-34]. Distinguishing between these two models has been challenging due to the overlapping nature of the early steps of HIV replication, differing methods used to assay uncoating, and the characteristics of defective or noninfectious virions possibly confounding results. Data from studies supporting both models suggest that a pore opens to destabilize the capsid integrity and then the hexameric CA lattice disassembles, but the time lag between these events is not clear [30, 34-38]. These models are also not necessarily mutually exclusive. A recent hypothesis that draws on both models proposes that the HIV capsid is destabilized in the cytoplasm, followed by additional loss of capsid at the nuclear pore $[12,39]$.

The primary viral factors involved with uncoating are the CA protein and the process of reverse transcription. Mutations in CA can decrease or increase the rate of uncoating which also decreases HIV infectivity, indicating that correct timing of uncoating is required for optimal HIV replication [7, 40-44]. Reverse transcription is also necessary for uncoating as inhibiting this process delays uncoating in cultured cell based, biochemical, and microscopy based uncoating assays [8-10, 30, 37, 38, 45]. Specifically, the generation of minus strand strong stop DNA, an early product of reverse transcription, is proposed to initiate uncoating [30,45]. Multiple cellular proteins have been found to play at least an indirect role in uncoating $[11,12,27]$. Disruption of the interaction between eukaryotic translation elongation factor $1 \mathrm{~A}(\mathrm{eEF} 1 \mathrm{~A})$ and reverse transcriptase delays uncoating, further highlighting the interplay between reverse transcription and uncoating [46]. Knockdown of proteins involved with microtubule trafficking can delay uncoating and impair cytoplasmic trafficking of viral complexes, including the motor proteins dynein and kinesin-1 Kif5B, kinesin-1 adaptor protein FEZ1, microtubule affinity-regulating kinase 2 (MARK2), dynein adaptor protein BICD2, and cytoskeletal regulatory proteins Dia1 and Dia2 [13-19]. In addition, knockdown of the nuclear pore protein NUP358 disrupts cytoplasmic trafficking, nuclear import, and uncoating $[13,19]$. The proteins BICD2, Dia1, Dia2, FEZ1, MARK2, and NUP358 also have been shown to directly bind to the capsid to mediate these effects, indicating a direct effect on uncoating $[13-15,18,19]$. Collectively these results suggest that uncoating must occur within the correct spatiotemporal context so that the viral complex can interact with cellular factors for productive HIV infection.

We have previously examined the kinetics of uncoating in owl monkey kidney (OMK) cells and HeLa cells using the CsA washout assay $[8,40]$. This assay utilizes the activity of the restriction factor TRIM-CypA to bind to the HIV capsid and then inhibit infectivity [8, 47]. Withdrawal of the drug cyclosporine A (CsA) is used to activate this TRIM-CypA restriction at various times post-infection, allowing the kinetics of uncoating to be revealed. The CsA washout assay is indirect in that relies on the activity of TRIM-CypA to detect uncoating rather than directly measuring the level of CA protein. However, fluorescence microscopy based uncoating assays and biochemical uncoating assays that directly detect the loss of CA have confirmed a similar timing, effect of reverse transcription, effect of CA mutations, and effect of cellular factors on uncoating [8-10, 16, 30, 38, 45, 48]. Therefore, while indirect the CsA washout assay provides a good monitor for the kinetics of successful uncoating in productively infected cells. An additional strength of the CsA washout assay is that it provides a direct correlation between uncoating and infectivity. In order for an uncoating event to be detected, the virus must successfully uncoat and integrate its DNA into the host cell DNA to establish productive infection. The majority of HIV virions that enter cells do not establish productive infection. The characteristics of these defective virions could bias or obscure results in uncoating studies where a large number of virions are surveyed without accounting for productive infection [29, 36-38, 48-51].

We previously used the CsA washout assay to examine the effect of different $C A$ mutations on uncoating in infected cells [40]. These CA mutations can affect the infection of nondividing cells, utilization of nuclear import pathways, integration site selection, and interaction with cellular proteins that facilitate HIV infection [13, 20-23, 48, 52, 53]. We found that the E45A and N74D mutations uncoated slower than wildtype virus, while the mutation A92E uncoated faster than wildtype in OMK cells [40]. We also observed differential uncoating kinetics for the mutant N74D in a HeLa cell line engineered to express TRIM-CypA [40]. These results suggest that cell type differences can impact uncoating kinetics. 
Given the possible role of cellular environment, here we have extended our analysis of uncoating to the human microglial cell lines CHME3 and C20 [54, 55]. Microglial cells are natural targets of HIV infection in humans and serve as a major viral reservoir in the central nervous system [56]. Infection and activation of microglial cells is proposed to be responsible for AIDS associated dementia and other neurocognitive defects observed in some AIDS patients [56]. There has also been recent interest to use cultured microglial cell models to study HIV latency [55]. Therefore, it is important to characterize HIV uncoating in this natural target of HIV infection. Given our previous experiments in OMK and HeLa cells, we examined the kinetics of uncoating, role of reverse transcription, and the effect of different CA mutants on the process of uncoating in these cultured microglial cell lines.

\section{Material and methods}

\section{Cell lines, viruses, and pharmaceuticals}

293 T cells were obtained from the American Type Culture Collection and were cultured in Dulbecco's Modified Eagle Media (Cellgro), 10\% fetal bovine serum (Atlanta Biologicals), and 1\% Pen/Strep/Glutamine (Gibco). CHME3 cell line was a gift M. Naghavi (Northwestern University $[54,57])$. The C20 cell line was a gift from D. Alvarez-Carbonell (Case Western Reserve University [55]). Prior to this study these cell lines were previously established from human microglia $[54,55]$. The stable cell lines CHME3-TC and C20-TC were made using an HA-tagged TRIM-CypA expressing retroviral vector (pLXSN-TRIMCypA) to infect CHME3 and C20 cells [58]. Neomycin resistant clonal cell lines (CHME3TC) or hygromycin resistant clonal cell lines (C20-TC) were screened for TRIM-CypA expression by western blot against HA and restriction activity. CHME3, CHME3-TC, C20 and C20-TC cells were cultured in Dulbecco's Modified Eagle Media (Cellgro), 5\% fetal bovine serum (Atlanta Biologicals), 1\% Pen/Strep/Glutamine (Gibco), and $1 \%$ sodium pyruvate (Gibco). All cell lines were maintained at $37{ }^{\circ} \mathrm{C}$ and $5 \% \mathrm{CO}_{2}$. VSV-g pseudotyped GFP reporter virus was produced by PEI transfection of $293 \mathrm{~T}$ cells with $6 \mu \mathrm{g}$ HIV-1 proviral plasmid HIV-GFP or proviral CA mutant plasmid and $4 \mu \mathrm{g}$ VSV-g expression plasmid. Virus was harvested $48 \mathrm{~h}$ post-transfection, purified through a $0.45 \mu \mathrm{m}$ filter, and stored at $-80^{\circ} \mathrm{C}$ until use. Cyclosporine A (Calbiochem) was prepared in ethanol and used at a final concentration of $2.5 \mu \mathrm{M}$. Nevirapine (NIH AIDS Research and Reference Reagent Program) was prepared in DMSO and used at a final concentration of $5 \mu \mathrm{M}$. Ammonium chloride was prepared in double-distilled water $\left(\mathrm{ddH}_{2} \mathrm{O}\right)$ and used at a final concentration of $10 \mu \mathrm{M}$.

\section{CsA washout assay}

The CsA washout assay was conducted as previously described [8, 59]. Briefly, CHME3-TC or C20-TC cells were plated in 96 well dishes at a density of 6000 cells /well, with each experimental or control reaction performed on triplicate wells. These cells were spinoculated with VSV-g pseudotyped HIV-GFP or CA mutant virus in the presence of CsA and $10 \mu \mathrm{g} / \mathrm{ml}$ polybrene for $1 \mathrm{~h}$ at $16^{\circ} \mathrm{C}$. After spinoculation, the inoculation media was exchanged for warm media, and CsA was washed out of the zero time point reaction by media exchange. CsA washout by media exchange continued at various times post-infection. The negative control for each time point was ethanol washout by media exchange. To examine the effect of reverse transcription on uncoating nevirapine was included in the inoculation media and washout media for the first $2 \mathrm{~h}$ of the experiment. After $2 \mathrm{~h}$ it was removed by media exchange on all reactions. Two days post-infection cells were harvested with $100 \mu$ ltrypsin and fixed by the addition of $100 \mu \mathrm{l}$ fix (4:1, 1X PBS: $10 \%$ paraformaldehyde). The percentage of GFP positive cells was determined by flow cytometry using the Accuri C6 flow cytometer, averaged for each triplicate reaction, and standard error was calculated. The percentage of infected cells in the ethanol control was subtracted from the CsA reaction at each washout time point to yield the percentage of infected cells over background. The data was normalized by setting the highest percentage of GFP positive cells to $100 \%$ for each virus or condition tested. The half-life of uncoating was determined by a best fit line through the two data points flanking 50\% uncoating and times were averaged from multiple independent experiments. For statistical analysis of the half-life of uncoating, the average half-life of uncoating was compared between the CA mutant virus and its corresponding wildtype CA control using an unpaired T-test.

\section{Viral fusion assay}

CHME3-TC and C20-TC cells were plated and spinoculated with HIV-GFP virus as described in the CsA washout assay. When inoculation media was exchanged for warm media, media containing CsA and the fusion inhibitor ammonium chloride was added to the zero time point reaction. Ammonium chloride addition continued by media exchange at various times post-infection. Controls included no treatment and continuous treatment with ammonium chloride. Two days post-infection, cells were harvested and the percentage of GFP positive cells was determined as in the CsA washout assay. The halflife of fusion was determined using a best fit line between the adjacent data points and times were averaged from five independent experiments. These assay were conducted in parallel with a CsA washout assay to 
directly compare the half-lives of viral fusion and uncoating.

\section{Nevirapine addition assay}

CHME3-TC cells were plated and spinoculated with HIV-GFP virus as described in the CsA washout assay. When inoculation media was exchanged for warm media, media containing CsA and the reverse transcriptase inhibitor nevirapine (NVP) was added to the zero time point reaction. NVP addition continued by media exchange at various times post-infection. Controls included continuous treatment with NVP and DMSO carrier control (no NVP treatment). Two days postinfection, cells were harvested and the percentage of GFP positive cells was determined as in the CsA washout assay. For each virus, infectivity at each timepoint was normalized by setting the percentage of infected cells in the no nevirapine control to $100 \%$. Data from three independent experiments was averaged for each virus. For statistical analysis of the progression of reverse transcription, an unpaired T-test was used to compare the percentage of GFP positive cells between each CA mutant virus and the corresponding wildtype CA control at each timepoint tested.

\section{CsA addition assay}

The parent CHME3 cell line used to generate CHME3TC cells was plated as described in the CsA washout assay. Cells were spinoculated with VSV-g pseudotyped HIV-GFP in the presence of $10 \mu \mathrm{g} / \mathrm{ml}$ polybrene for $1 \mathrm{~h}$ at $16{ }^{\circ} \mathrm{C}$. When this inoculation media was exchanged for warm media, media containing CsA or the carrier control ethanol was added to the zero time point reaction. CsA or ethanol addition continued by media exchange at various times post-infection. Controls included no treatment and continuous treatment for both the CsA and ethanol conditions. Cells were harvested and the percentage of GFP positive cells was determined as in the CsA washout assay. The ratio of infectivity was calculated by dividing the percentage of GFP positive cells in the CsA reaction by the percentage of GFP positive cells in the ethanol control reaction for each time point.

\section{Results}

\section{Kinetics of uncoating in microglial cells}

To examine the kinetics of uncoating in microglial cells, we performed the CsA washout assay [8, 47]. In this assay the HIV restriction factor TRIM-CypA is used to detect uncoating $[60,61]$. To restrict infection multiple TRIM-CypA proteins assemble in a lattice to bind the hexameric array of CA protein found in the assembled HIV-1 capsid [60, 62-64]. Therefore, TRIM-CypA will bind and inhibit the infectivity of coated viral complexes.
The drug cyclosporine A (CsA) prevents the binding of TRIM-CypA to the viral capsid in a reversible fashion $[47,60,61,65]$. In the CsA washout assay, cells are synchronously infected with a GFP reporter virus in the presence of CsA. When CsA is then removed at various times post-infection, TRIM-CypA can bind to any coated viral complexes and inhibit infectivity. However, viral complexes that have progressed through uncoating such that they are resistant to TRIM-CypA binding and restriction will still be able to infect the cell. Two days post-infection cells are harvested and flow cytometry is used to determine the percentage of infected cells at each time point. As only uncoated viral complexes can establish productive infection during TRIM-CypA restriction, this percentage of infected cells correlates to the percentage of uncoated virions at each timepoint [8].

We engineered the human microglial cell lines CHME3 and C20 to express TRIM-CypA so that the CsA washout assay could be used to study uncoating $[54,55]$. The CHME3 cell line, also present in the literature as HMC3, has been verified by a recent study and the ATCC as a human microglial cell line [57]. The C20 cell line has been more recently established from adult human cortical microglia and has been characterized as human microglial by morphology, surface marker expression, RNA expression profile, and sequencing [55]. CHME3 and C20 cells were infected with a retroviral vector encoding an HA-tagged version of owl monkey TRIM-CypA. A clonal stable cell line (CHME3-TC or C20-TC) was established which expressed TRIM-CypA protein at a sufficient level to restrict HIV replication. Treatment with the drug cyclosporine A (CsA) was able to relieve TRIM-CypA restriction in the CHME3-TC and C20-TC cell lines. To characterize uncoating the CsA washout assay was performed in CHME3-TC cells and C20-TC cells using the VSV-g pseudotyped GFP reporter virus HIV-GFP. In these assays, there was an increase in the percentage of uncoated viral complexes over time, which leveled off at $4-5 \mathrm{~h}$ post infection (Fig. 1a). In both cell lines, the majority of uncoating $(\sim 80 \%)$ occurred within the first $2 \mathrm{~h}$ of the experiment. The data were then normalized by setting the percentage of GFP positive cells where the curve levels off at 4 or $5 \mathrm{~h}$ to $100 \%$, allowing a half-life of uncoating or time of $50 \%$ uncoating to be calculated (Fig. 1a). The average half-life of uncoating was 52.46 min in CHME3-TC cells and $34.95 \mathrm{~min}$ in C20-TC cells (Table 1 ).

We have previously shown that alterations in the timing of viral fusion can impact the perceived rate of uncoating in the CsA washout assay [8, 40]. Viral fusion immediately precedes uncoating and the time for both viral fusion and uncoating is included in the half-life of uncoating as calculated above. Therefore, differences in the rate of viral fusion between CHME3-TC and C20- 

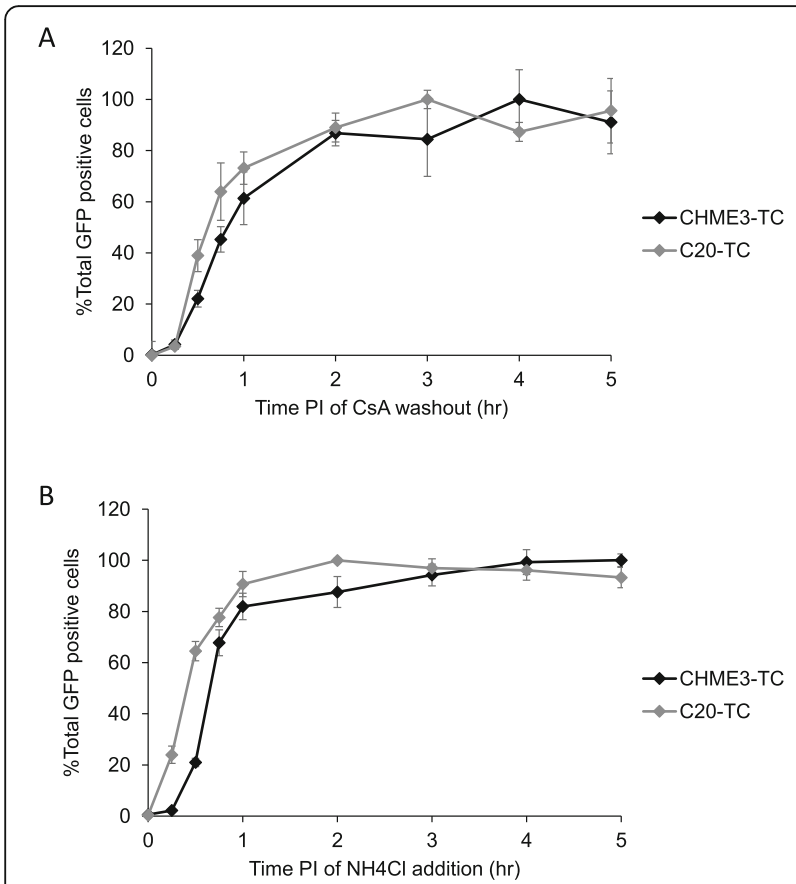

Fig. 1 Kinetics of uncoating and viral fusion in CHME3-TC and C20TC cells. CSA washout assays and viral fusion assays were performed in parallel to correlate the kinetics of uncoating and viral fusion in CHME3-TC and C20-TC cells. Shown is a representative experiment from five independent parallel experiments. Errors bars denote standard error among triplicate wells. a The uncoating kinetics were similar in both cell lines with the majority of virus uncoating within $2 \mathrm{~h}$ post-infection. $\mathbf{b}$ The kinetics of viral fusion were examined by the addition of ammonium chloride at various times post-infection to block viral fusion. In both cell lines the majority of virus fused within $1 \mathrm{~h}$ post-infection

TC cells could appear as different uncoating rates. To directly compare the kinetics of viral fusion and uncoating in both cell lines, we performed a series of viral fusion assays in parallel with CsA washout assays. For the viral fusion assay cells were spinoculated with HIV-GFP in the presence of CsA and then ammonium chloride was added to block VSV-g mediated viral fusion at time points corresponding to those in CsA washout assay (Fig. 1b). The average half-life of viral fusion was 43.84 min in CHME3-TC cells and 26.3 min in C20-TC cells (Table 1). After subtracting the half-life of viral fusion from the half-life of uncoating, both microglial cell lines displayed a similar normalized rate of uncoating of 8.6 min (Table 1).

\section{Effect of reverse transcription on uncoating kinetics}

To determine the effect of reverse transcription on uncoating kinetics in microglial cells, we performed the CsA washout assay using the non-nucleoside reverse transcriptase inhibitor nevirapine to block reverse transcription for the first $2 \mathrm{~h}$ of the assay. This $2 \mathrm{~h}$ nevirapine treatment delayed the process of uncoating in CHME3-TC and C20-TC cells as evidenced by a shift in the uncoating curve to the right compared to no treatment (Fig. 2). Inhibition of reverse transcription increased in the half-life of uncoating to $137.9 \mathrm{~min}$ in CHME3-TC cells and $136.4 \mathrm{~min}$ in C20-TC cells. In addition, there was a rapid increase in the percentage of uncoated viral complexes in the first hour after nevirapine removal, between the 2 to $3 \mathrm{~h}$ timepoints, in both cell lines (Fig. 2).

\section{Effect of CA mutations on uncoating}

We previously examined the uncoating of a panel of CA mutant viruses in OMK cells and found that the N74D, A92E, and E45A mutations altered the rate of uncoating compared to wildtype [40]. These mutants are of interest due to their involvement with viral cytoplasmic trafficking, nuclear import, integration, and interaction with cellular factors [13, 20-23, 48, 52, 53]. Therefore, we examined the effect of these mutations on uncoating in microglial cells. Using the CsA washout assay, the uncoating kinetics of each CA mutant was examined in parallel to the wildtype control HIV-GFP in CHME3-TC cells. All three mutants decreased the infectivity of HIV. By normalizing the data for each mutant independently, this decreased level of infectivity does not bias uncoating kinetics in the CsA washout assay. Compared to HIVGFP, the N74D mutation delayed the process of uncoating and increased the half-life of uncoating to $141 \mathrm{~min}$ compared to $62 \mathrm{~min}$ for wildtype (Fig. 3a). The E45A mutation also delayed the process of uncoating compared to wildtype, with an average half-life of $102 \mathrm{~min}$ compared to $50 \mathrm{~min}$ for wildtype (Fig. 3b). We also examined the uncoating of virus containing the R132T second site suppressor mutation which partially restores the infectivity of E45A mutant virus [66]. This E45A/R123T mutant virus uncoated with kinetics like wildtype, with an average half-life of uncoating of $39 \mathrm{~min}$ that was not statistically different from the average half-life of $43 \mathrm{~min}$ for wildtype in parallel experiments (Fig. 3c). Finally, A92E mutant virus had an uncoating half-life of $62 \mathrm{~min}$

Table 1 Half-lives of viral fusion and uncoating in CHME3-TC and C20-TC cells

\begin{tabular}{llll}
\hline Cell line & Fusion $(\mathrm{min})$ & Uncoating $(\mathrm{min})$ & Normalized uncoating (min) \\
\hline CHME3-TC & $43.84(\mathrm{SE}=8.02)$ & $52.46(\mathrm{SE}=8.05)$ & 8.62 \\
C20-TC & $26.30(\mathrm{SE}=1.85)$ & $34.95(\mathrm{SE}=5.62)$ & 8.65 \\
\hline
\end{tabular}

The average half-lives of viral fusion and uncoating were determined from 5 independent parallel experiments. SE denotes standard error 


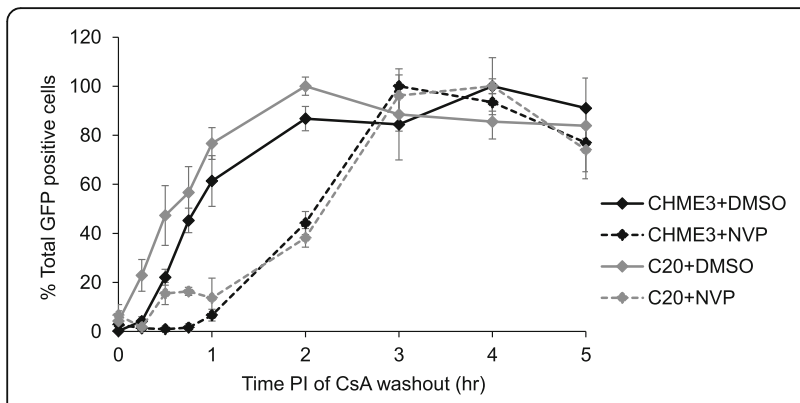

Fig. 2 Effect of reverse transcription on uncoating in CHME3-TC and C20-TC cells. The effect of reverse transcription on the process of uncoating in CHME3-TC and C20-TC cells was determined using a 2 $\mathrm{h}$ treatment of nevirapine (NVP) in the CsA washout assay.

Treatment with NVP delayed the process of uncoating and increased the average half-life of uncoating to $137.9 \mathrm{~min}$ in CHME3-TC cells and $136.4 \mathrm{~min}$ in C20-TC cells. Shown is a representative experiment from four independent experiments. Errors bars denote standard error among triplicate wells that was not statistically different from the $52 \mathrm{~min}$ for wildtype (Fig. 3d).

\section{Effect of CA mutations on reverse transcription}

Blocking reverse transcription delays uncoating in CHME3-TC cells and some CA mutations can disrupt reverse transcription (Fig. 2 [7]). Therefore, changes in the uncoating kinetics of the mutant viruses could be due to alterations in reverse transcription (Fig. 3). To examine the kinetics of reverse transcription for each mutant virus, we performed an addition assay with the non-nucleoside reverse transcriptase inhibitor nevirapine (NVP; Fig. 4). In this assay, cells were spinoculated with wildtype or CA mutant GFP reporter virus in the presence of CsA and then NVP was added at time points corresponding to those in CsA washout assay. At each timepoint, virus that had completed reverse transcription would be resistant to nevirapine and able to infect the cell. The data were normalized by setting the percentage of GFP positive cells in the DMSO carrier control to

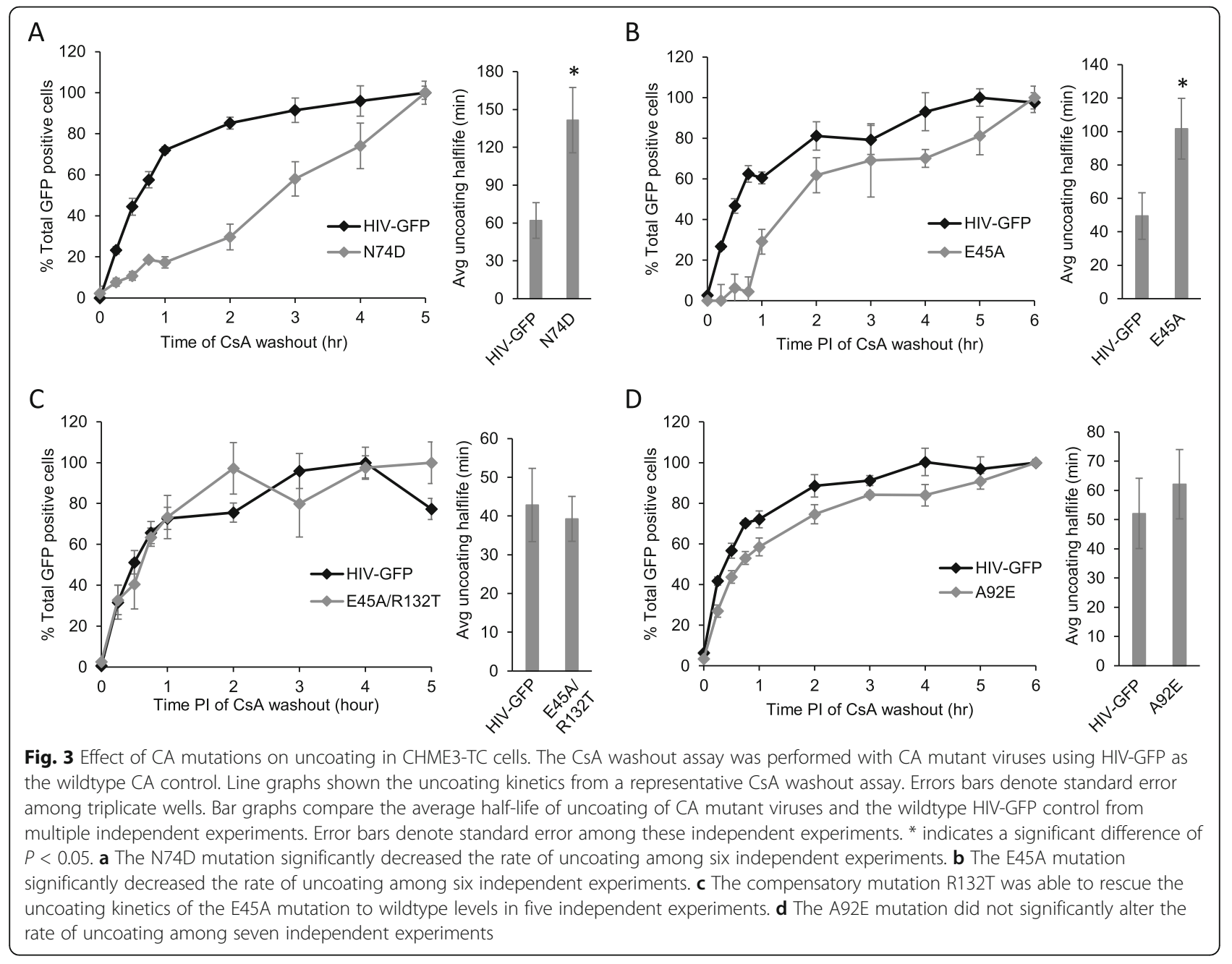




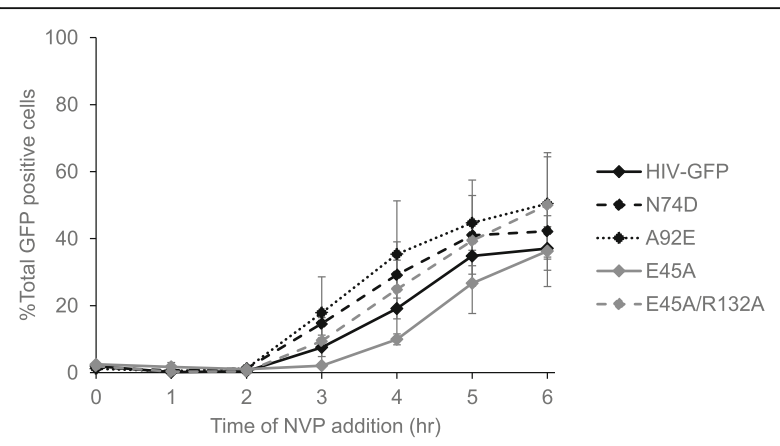

Fig. 4 Reverse transcription kinetics of CA mutant viruses in CHME3TC cells. Completion of reverse transcription was examined using a nevirapine addition assay. For each virus, infectivity at each timepoint was normalized to the DMSO carrier control. A statistically significant difference in reverse transcription compared to the HIVGFP control was not found at any timepoint. Shown is the average of three independent experiments. Error bars denote standard error among these independent experiments

$100 \%$. Some alterations in completion of reverse transcription were observed, with A92E seeming to reverse transcribe at the greatest rate and E45A at the slowest rate (Fig. 4). However, none of these differences were found to be statistically significant compared to the HIV-GFP wildtype control at any timepoint. Therefore, these data suggest that these CA mutations do not affect completion of reverse transcription at early time points post-infection in CHME3 cells.

\section{Effect of CsA in CHME3 cells}

Cyclosporine A is used in the CsA washout assay to control TRIM-CypA mediated restriction of infection. In HeLa cells CsA treatment has been shown to decrease the infectivity of N74D mutant virus, but not virus with a wildtype capsid [67]. In the CsA washout assay, the data is normalized for each virus independently, using the infectivity at 5 or $6 \mathrm{~h}$. However, if there was a differential effect of cyclosporin A on N74D virus over time, this effect could bias the normalized uncoating kinetics. Therefore, we examined the effect of CsA on wildtype and N74D infectivity in the parent CHME3 cell line over time by performing a CsA washout assay. Treatment with CsA decreased both wildtype and N74D infectivity at all time points examined compared to the ethanol control (Fig. 5). The magnitude of this decrease was determined by calculating ratio of infectivity for each time point examined (Table 2). In general, the CsA containing reactions exhibited $63-75 \%$ of the infected cells in the corresponding ethanol control (Table 2). This decrease was consistent across all time points examined and within a range of $70-75 \%$ during the first $2 \mathrm{~h}$ after infection when the majority of uncoating is observed (Fig. 4, Table 2).

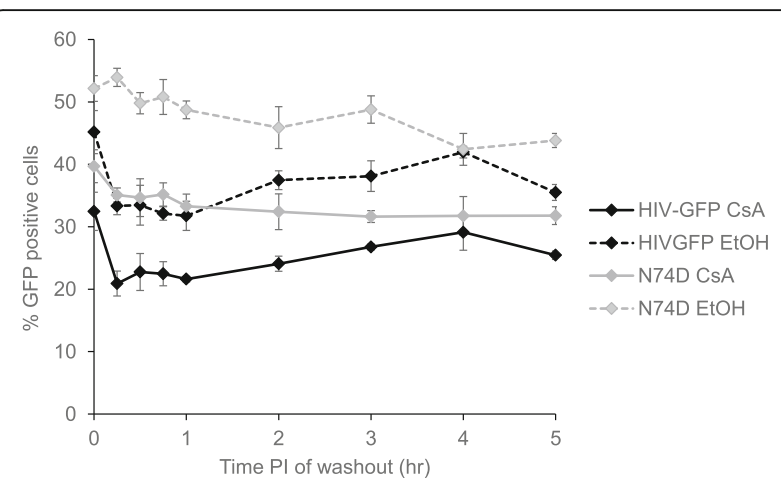

Fig. 5 Effect of CSA on infectivity in CHME3 cells. The effect of CSA on HIV-GFP and N74D infectivity in the parent CHME3 cell line was determined by comparing CsA washout and EtOH washout at times corresponding to the CSA washout assay. The presence of CSA decreases the infectivity of both HIV-GFP and N74D virus at all time points tested. Shown is a representative assay from three independent experiments. Error bars denote standard error among triplicate wells

\section{Discussion}

Here we have characterized HIV uncoating in the microglial cell lines CHME3 and C20. Similar to previous studies in owl monkey kidney (OMK) cells and HeLa cells, the half-life of uncoating was within an hour post infection and the majority of virus $(\sim 80 \%)$ uncoated within the first $2 \mathrm{~h}$ of infection (Fig. 1 and Table $1[8,40]$ ). When comparing the kinetics, uncoating in CHME3 cells had average half-life of 52.46 min which is intermediate between what was previously observed in HeLa cells $(36.8 \mathrm{~min})$ and OMK cells (64 min [40]). The average half-life of uncoating in C20 cells was 34.95 min which is similar to the halflife in HeLa cells [40]. As uncoating follows viral fusion, alterations in the rate of viral fusion in different cell lines could alter the perceived rate of uncoating. We have

Table 2 Ratio for the effect of CSA on HIV infectivity

\begin{tabular}{lll}
\hline $\begin{array}{l}\text { Time } \\
\text { (hr) }\end{array}$ & \multicolumn{2}{c}{ CsA/EtOH ratio } \\
\cline { 2 - 3 } HIV-GFP & N74D \\
\hline 0 & 0.72 & 0.76 \\
0.25 & 0.63 & 0.65 \\
0.5 & 0.68 & 0.70 \\
0.75 & 0.70 & 0.69 \\
1 & 0.68 & 0.68 \\
2 & 0.64 & 0.68 \\
3 & 0.70 & 0.65 \\
4 & 0.69 & 0.75 \\
5 & 0.72 & 0.73 \\
\hline
\end{tabular}

The magnitude of the effect of $\mathrm{CsA}$ was determined by calculating a ratio of infectivity (\%GPP positive cells with CsA treatment/\%GPP positive cells in EtOH carrier control) for each time point examined for HIV-GFP and N74D virus. In general, the CsA containing reactions exhibited $63-75 \%$ of the infected cells in the corresponding ethanol control 
previously observed this effect when characterizing the rate of uncoating of VSV-g pseudotyped virus and virus with wildtype HIV envelope in OMK cells, and the kinetics of uncoating in OMK and HeLa cells $[8,40]$. Once the half-life of viral fusion was subtracted, the uncoating halflife in both CHME3 and C20 cells was $8.6 \mathrm{~min}$ (Table 1). These results suggest that in these microglial cell lines the uncoating process is initiated fairly early after viral fusion, within $8 \mathrm{~min}$. These kinetics are most similar to HeLa cells where the difference in the average half-lives of viral fusion and uncoating was 8 min, while in OMK cells this difference was $45 \mathrm{~min}$ [40]. HeLa, CHME3, and C20 cells are all human cell lines, therefore it is likely they would display more similarities in uncoating compared to OMK cells.

We do not believe that expression levels of TRIMCypA protein could account for the differences in uncoating kinetics between CHME3, C20, and OMK cells. The CsA washout assay was conducted under conditions in which TRIM-CypA restriction was not saturated, meaning that in the absence of CsA viral infectivity was completely inhibited. TRIM proteins rapidly associate with incoming virus [47]. Under these non-saturating conditions a sufficient amount TRIMCypA protein was present to bind to the viral capsid and inhibit infectivity. The presence of additional TRIMCypA protein should not have an additive effect on restriction to alter the detection of coated viral complexes in the CsA washout assay. A more likely hypothesis is that differences in the kinetics of uncoating between the microglial cell lines and OMK cells are due to the presence or absence of cellular factors. Several cellular motor and trafficking proteins have been identified which affect HIV uncoating [13-19]. Differential expression or the differential ability of HIV capsid to interact with owl monkey cellular factors could account for the overall delayed uncoating kinetics in OMK cells compared to CHME3 and C20 cells.

The process of reverse transcription has been shown to have a complicated interplay with the process of uncoating. Similar to previous studies in OMK cells, inhibition of reverse transcription in CHME3 and C20 cells delayed the process of uncoating in the CsA washout assay (Fig. 2 [8]). This effect of reverse transcription on uncoating is consistent with multiple studies using a variety of uncoating assays $[8-10,15,30,38,45]$. In addition, there was a rapid increase in the percentage of uncoated virions in the first hour after the nevirapine treatment was removed, indicating that a large number of viral complexes initiated uncoating after reverse transcription was allowed to proceed (Fig. 2). This result supports the model where minus strand strong stop DNA initiates uncoating as this early reverse transcription product could readily be generated in the hour after nevirapine removal due to its short length [30,45]. Our results also concur with a recent study in which the fate of the capsid uncoating assay was used to examine the effect of reverse transcription on uncoating in CHME3 cells [15]. While this was not a kinetic analysis, inhibition of reverse transcription resulted in viral cores with increased amounts of CA protein at $3 \mathrm{~h}$ post-infection, indicating a delay in uncoating [15].

We next examined the effect of CA mutations on the process of uncoating. The N74D, E45A, and A92E mutants were chosen because they significantly altered the rate of uncoating in OMK cells in our previous study [40]. These mutants also can affect the infection of nondividing cells, utilization of nuclear import pathways, integration site selection, and interaction with cellular proteins that facilitate HIV infection [13, 20-23, 48, 52, 53]. Given the similarities in the kinetics of uncoating and effect of reverse transcription on uncoating in CHME3 and C20 cells, we tested these mutants only in the CHME3-TC cell line. N74D and E45A significantly altered the rate of uncoating compared to the parallel HIV-GFP control in CHME3 cells (Fig. 3). The effect of each mutation on uncoating was likely more severe than the data indicate because the CsA washout assay is based on infectivity. An infected cell in this assay indicates that the virus has successfully uncoated and then established a provirus to express the GFP reporter. Therefore, the uncoating kinetics of these mutants reveal the extent to which changes in uncoating can be tolerated while still resulting in productive infection.

Given that changes in the rate of reverse transcription can affect uncoating we tested progression of reverse transcription in the CA mutants. We found that these mutations did not significantly change the rate of reverse transcription at early timepoints post-infection in CHME3 cells (Fig. 4 [40]). Therefore, the alterations in uncoating for E45A and N74D virus were not due to alterations in reverse transcription. This result is similar to what was previously found in OMK cells for the E45A, N74D, and A92E mutations [40]. While some CA mutations have been shown to have altered capsid stability and changes in reverse transcription, the capsid mutations in this study provide a way to uncouple the process of uncoating from reverse transcription [7]. In previous experiments studying the effect of reverse transcription on uncoating in OMK cells, we found that each CA mutant virus had a similar delay in uncoating in response to nevirapine treatment [40]. Therefore, the effect of delaying uncoating in the CsA washout assay was dominant to the effect CA mutations. This result indicates that the contribution of reverse transcription to uncoating occurs before the effect of the N74D and E45A capsid mutations. Thus, these data also support the model by which the minus strand strong stop DNA initiates uncoating as this product is generated early in infection. 
While the N74D mutation delayed uncoating in CHME3 cells, there are differences in the magnitude of this change compared to our previous studies [40]. In CHME3 cells N74D mutant virus uncoated with a halflife of $141 \mathrm{~min}$ compared to a half-life of $62 \mathrm{~min}$ for wildtype in the parallel assays (Fig. 3a). This $\sim 2$-fold change in uncoating kinetics is most similar to results in HeLa cells, while in OMK cells the N74D mutation only increased that half-life of uncoating by $50 \%$ [40]. This mutation has also been shown to delay uncoating in fluorescence microscopy based uncoating assays [30, 35]. The N74D mutation causes HIV to utilize a different nuclear import pathway than that mediated by the importin- $\beta$ protein TNPO3, nucleocytoplasmic shuttle protein CPSF6, and nucleoporin NUP358 [20, 21, 23]. A study by Dharan et al. showed that knockdown of NUP358 and the microtubule kinesin-1 motor protein Kif5B delayed uncoating in HeLa cells using the fluorescence microscopy based in situ uncoating assay [13]. In this study the N74D mutation also prevented NUP358 and Kif5B from binding to the HIV capsid [13]. Therefore, a likely hypothesis is that the delayed uncoating kinetics of N74D mutant virus in CHME3 cells is due to the inability of this mutant to bind Kif5B or NUP358.

The E45A mutation produces a hyperstable capsid lattice with increased stiffness in atomic force microscopy assays [7, 68]. Like the N74D mutation, this mutation also results in the use of an alternate nuclear import pathway than that mediated by TNPO3, CPSF6, and NUP358 [20, 21]. In the CsA washout assay, the E45A mutation slowed the rate of uncoating in CHME3 cells with an increased half-life of $102 \mathrm{~min}$ compared to the wildtype control (Fig. 3b). This result is similar to our previous study, although there was a more modest delay in uncoating due to the E45A mutation in OMK cells [40]. E45A virus was also observed to uncoat slower than wildtype in fluorescence microscopy based uncoating assays $[36,38]$. To determine whether capsid stability or disrupted interaction with cellular factors was responsible for the delayed uncoating of E45A mutant virus, we examined the uncoating kinetics of the E45A/R123T double mutant virus. R132T is a second site suppressor mutation that was isolated from serial passage of E45A mutant virus in culture [66]. In this study, the R132T compensatory mutation partially rescued infectivity and nuclear import defects of E45A mutant virus [66]. However, E45A/R132T virus still had a hyperstable capsid [66]. Surprisingly, we found that E45A/R132T virus uncoated with kinetics similar to wildtype (Fig. 3c). Therefore, the delayed uncoating kinetics observed in E45A virus were not due to a hyperstable capsid. This result suggests that overall capsid stability may not be a good predictor of uncoating kinetics, in agreement with other studies [24, 40, 66, 69]. The E45 and R132 residues are distant from each other but are both located near the NTD-NTD interface. As widespread structural changes are not observed in E45A or R132T mutant viruses, the altered chemical natures of these side chains were proposed to account for the effects of the mutations on replication [66]. The R132T mutation may restore an interaction surface needed for uncoating, and disruption of this region by the E45A mutation would result in delayed uncoating. The E45 residue has not been tested for NUP358 or Kif5B binding like the N74 residue, but mutation at this location may prevent the association with NUP358, Kif5B, or other members of the canonical nuclear import pathway that may be involved with uncoating. Supporting this idea, Yang et al. showed that the $\mathrm{R} 132 \mathrm{~T}$ mutation restored the ability of E45A virus to bind the drug PF74 [66]. PF74 directly binds to the conical capsid at a site where the cellular factors CPSF6 and the nucleoporin NUP153 bind to mediate nuclear import [25, 70, 71].

Yang et al. also found that E45A/R132T virus had less capsid disassembly compared to wildtype in an in vitro uncoating assay which may seem to contradict our results [66]. In this assay viral capsid cores were purified from virus, diluted in buffer, and the extent of disassembly after 30 min was assessed by detecting the remaining intact cores. However, this in vitro uncoating assay did not expose the capsid to cellular factors which may be needed for uncoating. In the absence of cellular factors E45A/R132T virus likely uncoated slower than wildtype and with a similar efficiency as E45A virus due to its increased capsid stability. In the CsA washout assay E45A/ $\mathrm{R} 132 \mathrm{~T}$ virus uncoated at a rate that was not significantly different from wildtype because this assay is performed in cells which would allow interactions with cellular factors to impact uncoating (Fig. 3).

A92E displayed different behavior than what we have previously observed. In OMK cells, this mutation increased the rate of uncoating compared to wildtype [40]. However, in CHME3 cells this mutation did not significantly change uncoating kinetics (Fig. 3d). A92E has been implicated in infection of nondividing cells and altered use the cellular factor cyclophilin A for HIV infection $[48,52,53]$. Given the species difference between OMK and human cell lines, it is possible that A92E necessitates the use of different cellular factors, thus resulting in a differential effect of this mutation between cell lines.

Cyclophilin A (CypA) is an abundantly expressed cytoplasmic protein that binds the capsid to alter HIV replication. The exact role of CypA in viral replication is unclear as it may increase, decrease, or have no effect on infectivity depending on the cell line tested [21, 72-74]. In cells where cyclophilin A facilitates HIV infection it has been proposed to play a role in the processes of reverse transcription, uncoating, and nuclear import [21, 72-75]. 
CypA was able to modulate uncoating in a cell type dependent manner in the fate of the capsid assay and stabilize the capsid in an in vitro uncoating assay [74, 76]. Recently, CypA has been proposed to protect the capsid from restriction by human TRIM5 $\alpha$ in primary human blood cells [77]. In the CsA washout assay, CsA is used to prevent binding of TRIM-CypA to the capsid which will also prevent cellular CypA from binding to the viral capsid. Therefore, observations cannot be made about the effect of CypA on uncoating of wildtype or CA mutant virus using the CsA washout assay. However, CsA treatment has been shown to decrease infectivity of N74D virus by 2-3-fold in HeLa cells, whereas wildtype infectivity was slightly increased [67]. Because the uncoating of each virus is normalized independently, different sensitivities of wildtype and N74D virus to CsA could impact uncoating kinetics if this sensitivity changes over the time course of the assay. Therefore, we performed a CsA washout assay in the parent CHME3 cell line to determine the kinetics of CsA sensitivity in N74D and wildtype viruses. Compared to the carrier control ethanol, CsA treatment decreased the infectivity of both wildtype and N74D mutant virus in CHME3 cells (Fig. 4). This decrease was greater for wildtype virus compared to N74D mutant virus. However, these decreases in infectivity were consistently in the range of 63-75\% over the time course of the CsA washout assay and $70-75 \%$ in the first $2 \mathrm{~h}$ of the assay for both viruses (Table 2). Therefore, in CHME3 cells sensitivity to CsA should not bias the uncoating kinetics for wildtype or N74D virus as determined by the CsA washout assay.

We chose to use the CsA washout assay to study uncoating as this assay is fairly high throughput compared to other uncoating assays, while allowing the assessment of uncoating kinetics at multiple timepoints post-infection [27]. In addition, the CsA washout assay provides a direct correlation between uncoating and successful infection of the cell. As the majority of HIV virions that enter cells do not establish productive infection, the characteristics of these defective virions could bias or obscure results. The CsA washout assay is indirect in that relies on the activity of TRIM-CypA to detect uncoating. TRIM-CypA self-associates into a hexagonal lattice that binds the hexameric CA lattice, but it is not known how much of the intact viral capsid must be present for TRIM-CypA lattice formation and restriction $[63,64]$. In the CsA washout assay viral capsids may uncoat to varying extents in the cytoplasm before exposure to TRIM-CypA binding with the withdrawal of CsA. However, in a microscopy assay only intact capsids localized to the cytoplasmic bodies of the closely related TRIM family member rhesus TRIM $5 \alpha$, indicating that the majority of the capsid lattice may be required for TRIM protein binding [78]. Therefore, we propose that in the CsA washout assay TRIM-CypA binds intact capsids or viral complexes that have just started to uncoat. The CsA washout assay would then detect the initial destabilization of the capsid, an early step of uncoating. In support of this hypothesis, Mamede et al. observed uncoating kinetics similar to the CsA washout assay in live cell imaging assays [30]. In this study, uncoating was directly monitored by a green fluorescent protein fluid phase marker that localized to the interior of the conical capsid. Live cell fluorescence imaging tracked the loss of this marker at the initiation of uncoating and also determined which virions resulted in productive infection of the cell [30]. In addition, multiple studies using fluorescence microscopy and biochemical assays that directly detect the loss of CA have revealed a similar timing, effect of reverse transcription, effect of CA mutations, and effect of cellular factors on uncoating as in the CsA washout assay [8-10, 16, 38, 45, 48]. Therefore, while indirect the CsA washout assay provides a good monitor for the kinetics of successful uncoating in productively infected cells.

\section{Conclusions}

In summary, we have characterized uncoating in the human microglial cell lines CHME3 and C20 which are natural targets of HIV infection. Similar to our previous results in cell lines that are not natural targets of HIV infection (OMK and HeLa cells), uncoating initiated within the first hour of infection and was facilitated by reverse transcription. These results support the model in which uncoating is initiated by early steps of reverse transcription in the cytoplasm and proceeds during transport of the viral complex to the nucleus. The CA mutations N74D and E45A delayed HIV uncoating, while the compensatory mutation $\mathrm{R} 132 \mathrm{~T}$ was able to rescue the uncoating kinetics of E45A mutant virus despite still having a hyperstable capsid. Discrepancies between the uncoating kinetics of CA mutant viruses in OMK and CHME3 cells reveals the importance of cellular factors in the process of uncoating. Specifically, the E45A/R132T mutant virus suggests that disrupted interactions with cellular factors, rather than capsid stability, is responsible for the delayed uncoating kinetics seen in E45A mutant virus. Future studies aimed at identifying these factors will be important for understanding the process of uncoating in cells, the influence of uncoating on other early steps of HIV replication, and the development of interventions to disrupt these processes.

\section{Abbreviations \\ CA: Capsid protein; CsA: Cyclosporin A; CypA: Cyclophilin A; HIV: Human immunodeficiency virus; NVP: Nevirapine; OMK: Owl monkey kidney}

\section{Acknowledgements}

The stable cell line CHME3-TC was created by AEH during a postdoctoral fellowship in the laboratory of Dr. Mojgan Naghavi (Northwestern University) and we would like to thank her for allowing the use of this cell line and the 
parent CHME3 cell line. We would like to thank Dr. David Alvarez-Carbonell (Case Western Reserve University) for the C20 microglial cell line and Dr. Chris Aiken (Vanderbilt University) for the HIV-GFP wildtype and CA mutant proviral plasmids. The following reagent was obtained through the NIH AIDS Reagent Program, Division of AIDS, NIAID, NIH: Nevirapine.

\section{Authors' contributions}

ZI performed experiments and analyzed data for the viral fusion assays. MT established the C20-TC line and performed initial uncoating and viral fusion assays in this cell line. GO performed initial uncoating assays with the E45A and E45A/R132T viruses. RM performed initial viral fusion assays in the CHME3-TC cell line. AEH performed uncoating, viral fusion, and reverse transcription experiments and wrote the manuscript. All authors read and approved the final manuscript.

\section{Funding}

This work was supported by a Faculty Research Grant (Missouri State University) and Summer Faculty Fellowship (Missouri State University) to AEH, a Graduate Research Funding award (Missouri State University) to MT, and Honors Research Funding award (Missouri State University) to GO. These funding sources had no role in the design of the study; the collection, analysis, and interpretation of data; or in writing of this manuscript.

\section{Availability of data and materials}

All data generated or analyzed during this study are included in this published article [and its supplementary information files].

\section{Ethics approval and consent to participate}

Not applicable.

\section{Consent for publication}

Not applicable.

\section{Competing interests}

The authors declare that they have no competing interests.

Received: 6 December 2019 Accepted: 21 February 2020 Published online: 06 March 2020

\section{References}

1. Briggs JAG, Simon MN, Gross I, Kräusslich H-G, Fuller SD, Vogt VM, et al. The stoichiometry of Gag protein in HIV-1. Nat Struct Mol Biol. 2004;11:672-5.

2. Ganser BK, Li S, Klishko VY, Finch JT, Sundquist WI. Assembly and analysis of conical models for the HIV-1 core. Science. 1999;283:80-3.

3. Ganser-Pornillos BK, Cheng A, Yeager M. Structure of full-length HIV-1 CA: a model for the mature capsid lattice. Cell. 2007;131:70-9.

4. McDonald D, Vodicka MA, Lucero G, Svitkina TM, Borisy GG, Emerman M, et al. Visualization of the intracellular behavior of HIV in living cells. J Cell Biol. 2002;159:441-52.

5. Arhel N, Genovesio A, Kim K-A, Miko S, Perret E, Olivo-Marin J-C, et al. Quantitative four-dimensional tracking of cytoplasmic and nuclear HIV-1 complexes. Nat Methods. 2006;3:817-24.

6. Sabo Y, Walsh D, Barry DS, Tinaztepe S, de Los Santos K, Goff SP, et al. HIV-1 induces the formation of stable microtubules to enhance early infection. Cell Host Microbe. 2013;14:535-46.

7. Forshey BM, von Schwedler U, Sundquist WI, Aiken C. Formation of a human immunodeficiency virus type 1 core of optimal stability is crucial for viral replication. J Virol. 2002;76:5667-77.

8. Hulme AE, Perez O, Hope TJ. Complementary assays reveal a relationship between HIV-1 uncoating and reverse transcription. Proc Natl Acad Sci U S A. 2011;108:9975-80.

9. Yang $Y$, Fricke T, Diaz-Griffero F. Inhibition of reverse transcriptase activity increases stability of the HIV-1 core. J Virol. 2013;87:683-7.

10. Kutluay SB, Perez-Caballero D, Bieniasz PD. Fates of retroviral core components during unrestricted and TRIM5-restricted infection. PLoS Pathog. 2013;9:e1003214

11. Dharan A, Campbell EM. Role of microtubules and microtubule-associated proteins in HIV-1 infection. J Virol. 2018;92:e00085-18. https:/doi.org/10.1128/JVI. 00085-18.

12. Rawle DJ, Harrich D. Toward the "unravelling" of HIV: Host cell factors involved in HIV-1 core uncoating. PLoS Pathog. 2018;14:e1007270.
13. Dharan A, Talley S, Tripathi A, Mamede Jl, Majetschak M, Hope TJ, et al. KIF5B and Nup358 cooperatively mediate the nuclear import of HIV-1 during Infection. PLoS Pathog. 2016;12:e1005700.

14. Malikov V, Naghavi MH. Localized phosphorylation of a kinesin-1 adaptor by a capsid-associated kinase regulates HIV-1 motility and uncoating. Cell Rep. 2017;20:2792-9

15. Delaney MK, Malikov V, Chai Q, Zhao G, Naghavi MH. Distinct functions of diaphanous-related formins regulate HIV-1 uncoating and transport. Proc Natl Acad Sci U S A. 2017;114:E6932-41.

16. Lukic Z, Dharan A, Fricke T, Diaz-Griffero F, Campbell EM. HIV-1 uncoating is facilitated by dynein and kinesin 1. J Virol. 2014;88:13613-25.

17. Pawlica P, Berthoux L. Cytoplasmic dynein promotes HIV-1 uncoating. Viruses. 2014;6:4195-211.

18. Dharan A, Opp S, Abdel-Rahim O, Keceli SK, Imam S, Diaz-Griffero F, et al. Bicaudal D2 facilitates the cytoplasmic trafficking and nuclear import of HIV1 genomes during infection. Proc Natl Acad Sci U S A. 2017;114:E10707-16.

19. Malikov V, da Silva ES, Jovasevic V, Bennett G, de Souza Aranha Vieira DA, Schulte B, et al. HIV-1 capsids bind and exploit the kinesin-1 adaptor FEZ1 for inward movement to the nucleus. Nat Commun. 2015:6:6660.

20. Lee K, Ambrose Z, Martin TD, Oztop I, Mulky A, Julias JG, et al. Flexible use of nuclear import pathways by HIV-1. Cell Host Microbe. 2010;7:221-33.

21. Schaller T, Ocwieja KE, Rasaiyaah J, Price AJ, Brady TL, Roth SL, et al. HIV-1 capsid-cyclophilin interactions determine nuclear import pathway, integration targeting and replication efficiency. PLoS Pathog. 2011;7: e1002439.

22. Koh Y, Wu X, Ferris AL, Matreyek KA, Smith SJ, Lee K, et al. Differential effects of human immunodeficiency virus type 1 capsid and cellular factors nucleoporin 153 and LEDGF/p75 on the efficiency and specificity of viral DNA integration. J Virol. 2013;87:648-58.

23. Matreyek KA, Engelman A. The requirement for nucleoporin NUP153 during human immunodeficiency virus type 1 infection is determined by the viral capsid. J Virol. 2011:85:7818-27.

24. Hulme AE, Kelley Z, Foley D, Hope TJ. Complementary assays reveal a low level of CA associated with viral complexes in the nuclei of HIV-1-infected cells. J Virol. 2015;89:5350-61.

25. Peng K, Muranyi W, Glass B, Laketa V, Yant SR, Tsai L, et al. Quantitative microscopy of functional HIV post-entry complexes reveals association of replication with the viral capsid. Elife. 2014:3:e04114.

26. Stultz RD, Cenker JJ, McDonald D. Imaging HIV-1 genomic DNA from entry through productive infection. J Virol. 2017;91:e00034-17. https:/doi.org/10.1128/ Jl.00034-17.

27. Campbell EM, Hope TJ. HIV-1 capsid: the multifaceted key player in HIV-1 infection. Nat Rev Microbiol. 2015;13:471-83.

28. Perez-Caballero D, Hatziioannou T, Zhang F, Cowan S, Bieniasz PD. Restriction of human immunodeficiency virus type 1 by TRIM-CypA occurs with rapid kinetics and independently of cytoplasmic bodies, ubiquitin, and proteasome activity [Internet]. J Virol. 2005;79:15567-72. https://doi.org/10.1128/jvi.79.24.15567-15572.2005.

29. Xu H, Franks T, Gibson G, Huber K, Rahm N, Strambio De Castillia C, et al. Evidence for biphasic uncoating during HIV-1 infection from a novel imaging assay. Retrovirology. 2013;10:70.

30. Mamede Jl, Cianci GC, Anderson MR, Hope TJ. Early cytoplasmic uncoating is associated with infectivity of HIV-1. Proc Natl Acad Sci U S A. 2017;114: E7169-78.

31. Arhel NJ, Souquere-Besse S, Munier S, Souque P, Guadagnini S, Rutherford $\mathrm{S}$, et al. HIV-1 DNA Flap formation promotes uncoating of the preintegration complex at the nuclear pore. EMBO J. 2007;26:3025-37.

32. Lahaye X, Satoh T, Gentili M, Cerboni S, Conrad C, Hurbain I, et al. The capsids of HIV-1 and HIV-2 determine immune detection of the viral CDNA by the innate sensor CGAS in dendritic cells. Immunity. 2013:39:1132-42

33. Rasaiyaah J, Tan CP, Fletcher AJ, Price AJ, Blondeau C, Hilditch L, et al. HIV-1 evades innate immune recognition through specific cofactor recruitment. Nature. 2013;503:402-5.

34. Burdick RC, Delviks-Frankenberry KA, Chen J, Janaka SK, Sastri J, Hu $\mathrm{W}-\mathrm{S}$, et al. Dynamics and regulation of nuclear import and nuclear movements of HIV-1 complexes. PLoS Pathog. 2017;13:e1006570.

35. Francis AC, Melikyan GB. Single HIV-1 Imaging Reveals Progression of Infection through CA-Dependent Steps of Docking at the Nuclear Pore, Uncoating, and Nuclear Transport. Cell Host Microbe. 2018;23: 536-48.e6. 
36. Márquez CL, Lau D, Walsh J, Shah V, McGuinness C, Wong A, et al. Kinetics of HIV-1 capsid uncoating revealed by single-molecule analysis. Elife. 2018;7: e34772. https://doi.org/10.7554/eLife.34772.

37. Da Silva Santos C, Tartour K, Cimarelli A. A novel entry/uncoating assay reveals the presence of at least two species of viral capsids during synchronized HIV-1 infection. PLoS Pathog. 2016;12:e1005897.

38. Francis AC, Marin M, Shi J, Aiken C, Melikyan GB. Time-resolved imaging of single HIV-1 uncoating in vitro and in living cells. PLoS Pathog. 2016;12: e1005709.

39. Francis AC, Melikyan GB. Live-cell imaging of early steps of single HIV-1 infection. Viruses. 2018;10:e275. https://doi.org/10.3390/v10050275.

40. Hulme AE, Kelley Z, Okocha EA, Hope TJ. Identification of capsid mutations that alter the rate of HIV-1 uncoating in infected cells. J Virol. 2015;89:643-51.

41. von Schwedler UK, Stray KM, Garrus JE, Sundquist WI. Functional surfaces of the human immunodeficiency virus type 1 capsid protein. J Virol. 2003;77: 5439-50.

42. Fitzon T, Leschonsky B, Bieler K, Paulus C, Schröder J, Wolf $H$, et al. Proline residues in the HIV-1 NH2-terminal capsid domain: structure determinants for proper core assembly and subsequent steps of early replication. Virology. 2000;268:294-307.

43. Tang S, Murakami T, Cheng N, Steven AC, Freed EO, Levin JG. Human immunodeficiency virus type $1 \mathrm{~N}$-terminal capsid mutants containing cores with abnormally high levels of capsid protein and virtually no reverse transcriptase. J Virol. 2003;77:12592-602.

44. Tang S, Murakami T, Agresta BE, Campbell S, Freed EO, Levin JG. Human immunodeficiency virus type $1 \mathrm{~N}$-terminal capsid mutants that exhibit aberrant core morphology and are blocked in initiation of reverse transcription in infected cells. J Virol. 2001;75:9357-66.

45. Cosnefroy O, Murray PJ, Bishop KN. HIV-1 capsid uncoating initiates after the first strand transfer of reverse transcription. Retrovirology. 2016;13:58.

46. Rawle DJ, Li D, Swedberg JE, Wang L, Soares DC, Harrich D. HIV-1 uncoating and reverse transcription require eEF1A binding to surface-exposed acidic residues of the reverse transcriptase thumb domain. MBio. 2018;9:e0031618. https://doi.org/10.1128/mBio.00316-18.

47. Perez-Caballero D, Hatziioannou T, Zhang F, Cowan S, Bieniasz PD. Restriction of human immunodeficiency virus type 1 by TRIM-CypA occurs with rapid kinetics and independently of cytoplasmic bodies, ubiquitin, and proteasome activity. J Virol. 2005;79:15567-72.

48. Yamashita M, Perez O, Hope TJ, Emerman M. Evidence for direct involvement of the capsid protein in HIV infection of nondividing cells. PLoS Pathog. 2007;3:1502-10.

49. Yang Y, Luban J, Diaz-Griffero F. The fate of HIV-1 capsid: a biochemical assay for HIV-1 uncoating. Methods Mol Biol. 2014;1087:29-36.

50. Burdick RC, Hu W-S, Pathak VK. Nuclear import of APOBEC3F-labeled HIV-1 preintegration complexes. Proc Natl Acad Sci U S A. 2013;110:E4780-9.

51. Shah VB, Aiken C. In vitro uncoating of HIV-1 cores. J Vis Exp. 2011. https:// doi.org/10.3791/3384.

52. Qi M, Yang R, Aiken C. Cyclophilin A-dependent restriction of human immunodeficiency virus type 1 capsid mutants for infection of nondividing cells. J Virol. 2008:82:12001-8

53. Ylinen LMJ, Schaller T, Price A, Fletcher AJ, Noursadeghi M, James LC, et al. Cyclophilin A levels dictate infection efficiency of human immunodeficiency virus type 1 capsid escape mutants A92E and G94D. J Virol. 2009:83:2044-7.

54. Janabi N, Peudenier S, Héron B, Ng KH, Tardieu M. Establishment of human microglial cell lines after transfection of primary cultures of embryonic microglial cells with the SV40 large T antigen. Neurosci Lett. 1995;195:105-8.

55. Garcia-Mesa Y, Jay TR, Checkley MA, Luttge B, Dobrowolski C, Valadkhan S, et al. Immortalization of primary microglia: a new platform to study HIV regulation in the central nervous system. J Neurovirol. 2017;23:47-66.

56. González-Scarano F, Martín-García J. The neuropathogenesis of AIDS. Nat Rev Immunol. 2005;5:69-81.

57. Dello Russo C, Cappoli N, Coletta I, Mezzogori D, Paciello F, Pozzoli G, et al. The human microglial HMC3 cell line: where do we stand? A systematic literature review. J Neuroinflammation. 2018;15:259.

58. Mamede JI, Sitbon M, Battini J-L, Courgnaud V. Heterogeneous susceptibility of circulating SIV isolate capsids to HIV-interacting factors. Retrovirology. 2013;10:77.

59. Hulme AE, Hope TJ. The cyclosporin A washout assay to detect HIV-1 uncoating in infected cells. Methods Mol Biol. 2014;1087:37-46.
60. Sayah DM, Sokolskaja E, Berthoux L, Luban J. Cyclophilin A retrotransposition into TRIM5 explains owl monkey resistance to HIV-1. Nature. 2004:430:569-73.

61. Nisole S, Lynch C, Stoye JP, Yap MW. A Trim5-cyclophilin A fusion protein found in owl monkey kidney cells can restrict HIV-1. Proc Natl Acad Sci U S A. $2004 ; 101: 13324-8$

62. Forshey BM, Shi J, Aiken C. Structural requirements for recognition of the human immunodeficiency virus type 1 core during host restriction in owl monkey cells. J Virol. 2005;79:869-75.

63. Li Y-L, Chandrasekaran V, Carter SD, Woodward CL, Christensen DE, Dryden KA, et al. Primate TRIM5 proteins form hexagonal nets on HIV-1 capsids. Elife. 2016:5:e16269. https://doi.org/10.7554/eLife.16269.

64. Wagner JM, Christensen DE, Bhattacharya A, Dawidziak DM, Roganowicz MD, Wan Y, et al. General model for retroviral capsid pattern recognition by TRIM5 proteins. J Virol. 2018. https://doi.org/10.1128/JVI.01563-17.

65. Towers GJ, Hatziioannou T, Cowan S, Goff SP, Luban J, Bieniasz PD. Cyclophilin A modulates the sensitivity of HIV-1 to host restriction factors. Nat Med. 2003;9:1138-43.

66. Yang R, Shi J, Byeon I-JL, Ahn J, Sheehan JH, Meiler J, et al. Second-site suppressors of HIV-1 capsid mutations: restoration of intracellular activities without correction of intrinsic capsid stability defects. Retrovirology. 2012;9: 30 .

67. Ambrose Z, Lee K, Ndjomou J, Xu H, Oztop I, Matous J, et al. Human immunodeficiency virus type 1 capsid mutation N74D alters cyclophilin A dependence and impairs macrophage infection. J Virol. 2012;86:4708-14.

68. Ramalho R, Rankovic S, Zhou J, Aiken C, Rousso I. Analysis of the mechanical properties of wild type and hyperstable mutants of the HIV-1 capsid. Retrovirology. 2016;13:17.

69. Shi J, Zhou J, Shah VB, Aiken C, Whitby K. Small-molecule inhibition of human immunodeficiency virus type 1 infection by virus capsid destabilization. J Virol. 2011;85:542-9.

70. Matreyek KA, Yücel SS, Li X, Engelman A. Nucleoporin NUP153 phenylalanine-glycine motifs engage a common binding pocket within the HIV-1 capsid protein to mediate lentiviral infectivity. PLoS Pathog. 2013;9: e1003693.

71. Price AJ, Jacques DA, McEwan WA, Fletcher AJ, Essig S, Chin JW, et al. Host cofactors and pharmacologic ligands share an essential interface in HIV-1 capsid that is lost upon disassembly. PLoS Pathog. 2014;10:e1004459.

72. De laco A, Luban J. Cyclophilin A promotes HIV-1 reverse transcription but its effect on transduction correlates best with its effect on nuclear entry of viral cDNA. Retrovirology. 2014;11:11.

73. Braaten D, Franke EK, Luban J. Cyclophilin A is required for an early step in the life cycle of human immunodeficiency virus type 1 before the initiation of reverse transcription. J Virol. 1996;70:3551-60.

74. Shah VB, Shi J, Hout DR, Oztop I, Krishnan L, Ahn J, et al. The host proteins transportin SR2/TNPO3 and cyclophilin A exert opposing effects on HIV-1 uncoating. J Virol. 2013;87:422-32.

75. Yin L, Braaten D, Luban J. Human immunodeficiency virus type 1 replication is modulated by host cyclophilin A expression levels. J Virol. 1998;72:6430-6.

76. Li Y, Kar AK, Sodroski J. Target cell type-dependent modulation of human immunodeficiency virus type 1 capsid disassembly by cyclophilin A. J Virol. 2009:83:10951-62.

77. Kim K, Dauphin A, Komurlu S, McCauley SM, Yurkovetskiy L, Carbone C, et al. Cyclophilin A protects HIV-1 from restriction by human TRIM5a. Nat Microbiol. 2019. https://doi.org/10.1038/s41564-019-0592-5.

78. Yu Z, Dobro MJ, Woodward CL, Levandovsky A, Danielson CM, Sandrin V, et al. Unclosed HIV-1 capsids suggest a curled sheet model of assembly. J Mol Biol. 2013;425:112-23.

\section{Publisher's Note}

Springer Nature remains neutral with regard to jurisdictional claims in published maps and institutional affiliations. 